\title{
ASSOCIAÇÃO ENTRE LOMBALGIA CRÔNICA E DEPRESSÃO
}

Rafael de Oliveira Pena Neto, Adrielle Andrade Pugas, Marjori Leiva Camparoto

Universidade do Oeste Paulista - UNOESTE, Curso de Medicina, Presidente Prudente, SP. e-mail: rafaelpneto@hotmail.com

\section{RESUMO}

A lombalgia com duração superior a 7-12 semanas é considerada crônica. Essa condição é reconhecida como um problema comum e que demanda altos custos em todo o mundo. É comum em pacientes portadores de dores lombares crônicas, a presença de depressão, ansiedade e distúrbios do sono. Embora não esteja claro que lombalgia crônica seja a causa base de depressão, é clara a associação entre elas. Esse estudo tem como objetivo identificar a influência da lombalgia crônica para o desenvolvimento de depressão e a frequência em que ocorre depressão nesses pacientes. A metodologia escolhida foi a revisão integrativa. Ao todo nove artigos foram selecionados. Sete estudos comprovaram a existência da relação entre lombalgia crônica e depressão. Nesses estudos podem-se encontrar resultados que variam de $4 \%$ a 69,7\%. A promoção de saúde e prevenção da instalação das patologias crônicas de coluna, através de medidas simples de educação da população é a melhor forma de prevenção da instalação do quadro crônico.

Palavras-chave: Lombalgia crônica, Depressão, Ansiedade, Qualidade de Vida, Promoção de Saúde.

\section{ASSOCIATION BETWEEN CHRONIC LOW BACK PAIN AND DEPRESSION}

\begin{abstract}
The low back pain with more 7-12 weeks is considered chronic. This condition is recognized as a common problem and demands high costs all around the world. It is common in patients with chronic low back pain, the presence of depression, anxiety and sleep disorders. Although it's unclear that chronic low back pain is the underlying cause of depression, there is a clear association between them. This study aims to identify the influence of chronic low back pain for the development of depression and how often depression occurs in these patients. The methodology chosen was the integrative review. Altogether nine articles were selected. Seven studies have proven the existence of the relationship between chronic low back pain and depression. In these studies can be found results ranging from $4 \%$ to $69.7 \%$. Promoting health and preventing the onset of chronic column pathologies through simple measures of public education is the best form of prevention of chronic condition installation.
\end{abstract}

Keywords: Chronic low back pain, Depression, Anxiety, Quality of life, Health Promotion.

\section{INTRODUÇÃO}

Define-se dor lombar como uma condição clínica de dor na parte inferior da coluna vertebral, de intensidade moderada ou intensa. Essa dor pode ter um caráter agudo ou tornar-se crônico ${ }^{1}$. Há dois tipos de lombalgias: específicas e as inespecíficas. Nas lombalgias específicas é possível identificar a causa da dor, sendo elas intrínsecas ou extrínsecas ${ }^{2}$. Em alguns casos, não são encontrados uma causa para o quadro álgico, denominando-se lombalgia inespecífica.
A lombalgia com duração superior a 7-12 semanas é considerada crônica ${ }^{3}$. Essa condição é reconhecida como um problema comum e que demanda altos custos em todo o mundo, onde apesar dos grandes gastos com pesquisas e tratamentos, a extensão do problema permanece inalterado ${ }^{4}$.

Estima-se que as dores lombares crônicas atinjam cerca de 5\%-30\% dos adultos, se tornando um grande problema de saúde pública ${ }^{5,6}$. $O$ alto índice de lombalgias se deve a inúmeros fatores e vem crescido, principalmente, 
devido a modificação dos ambientes de trabalho e ao aumento da expectativa de vida $^{7,8}$. Relacionados ao trabalho pode-se incluir a insatisfação com o trabalho, a postura de trabalho estática, o levantamento repetido de peso e o estresse ${ }^{7}$. Já o envelhecimento, naturalmente, desencadeia um processo de desgaste da coluna, que associado a fatores hereditários determinam a presença ou não de dor lombar e o nível da dor ${ }^{8}$.

As lombalgias crônicas também trazem prejuízos econômicos e psicossociais. No Brasil, as doenças da coluna representam a primeira causa de pagamento do auxílio-doença e a terceira causa de aposentadoria por invelidez ${ }^{9}$. Outra consequências como a deterioração da saúde geral, diminuição da participação e contato social, irritação, ansiedade e depressão, também são de importante destaque ${ }^{9,10}$.

Diante disso, surge a seguinte questão: qual a influência da lombalgia crônica para o desenvolvimento de depressão e qual a frequência em que ocorre depressão nesses pacientes?

Como visto, há uma grande incidência de dores lombares crônicas na população, assim como a correlação com depressão nesses pacientes. Assim, esse estudo tem como objetivo identificar a influência da lombalgia crônica para o desenvolvimento de depressão e a frequência em que ocorre depressão nesses pacientes.

\section{METODOLOGIA}

A metodologia escolhida para o alcance do objetivo foi a revisão integrativa. Esta surgiu devido a crescendo complexidade e quantidade de novas informações nas áreas da saúde ${ }^{13,14}$.

A construção do trabalho foi desenvolvida seguindo seis fases: elaboração da pergunta norteadora, busca ou amostragem na literatura, coleta de dados, análise crítica dos estudos incluídos, discussão dos resultados e apresentação da revisão integrativa ${ }^{14}$.

Foram formuladas as seguintes questões para guiar a revisão integrativa: qual a influência da lombalgia crônica para o desenvolvimento de depressão e qual a frequência em que ocorre depressão nesses pacientes?

O levantamento bibliográfico foi feito através da utilização das seguintes bases de pesquisa de artigos científicos: SciELO (Scienftific Electronic Library Online), BVS (Biblioteca Virtual em Saúde) e PubMed. As palavras-chave utilizadas foram: "Lombalgia", "Depressão" e "Qualidade de vida".

Os artigos foram inclusos nesta revisão integrativa seguindo os seguintes critérios de inclusão: artigos publicados em português e inglês; textos completos disponíveis nas bases de dados; publicação entre 2010 e 2015; artigos com a temática referente ao objetivo da revisão integrativa. Os critérios de exclusão utilizados foram: artigos publicados antes de 2010; artigos que divergiam da proposta do estudo.

Para síntese e análise dos artigos selecionados foi usado um quadro desenvolvido propriamente para esse fim, que contêm alguns aspectos importantes para alcançar o objetivo do trabalho. O quadro é composto por: nome dos autores, objetivo, tamanho da amostra, resultados, recomendações/conclusões ${ }^{15}$.

A análise dos artigos para construção dessa revisão integrativa foi realizada de forma descritiva, possibilitando reunir o conhecimento produzido por eles e dessa forma, atingir o objetivo que esse modele propõe.

\section{RESULTADOS}

Foram encontrados 1574 artigos na busca nas bases de dados. 1179 no PubMed, 3 na Scientific Eletronic Library Online (SciELO), 392 Biblioteca Virtual em Saúde (BVS). Após a seleção por títulos, publicação entre 2010 e 2015 e artigos completos disponíveis restaram 9 artigos.

\begin{tabular}{|c|c|c|c|c|c|}
\hline $\begin{array}{l}\text { Nome dos } \\
\text { autores }\end{array}$ & Objetivo & & Amostra & Resultados & $\begin{array}{l}\text { Recomendações/ } \\
\text { Conclusões }\end{array}$ \\
\hline $\begin{array}{l}\text { CASTRO, M. M. } \\
\text { C; } \\
\text { DALTRO, C. }\end{array}$ & $\begin{array}{l}\text { Avaliar } \\
\text { transtornos } \\
\text { sono e } \\
\text { prevalência } \\
\text { sintomas } \\
\text { ansiedade }\end{array}$ & $\begin{array}{r}\text { os } \\
\text { de } \\
\text { a } \\
\text { dos } \\
\text { de } \\
\text { e }\end{array}$ & 400 & $\begin{array}{l}\text { A média de idade dos } \\
\text { pacientes foi } 45,6 \pm 11,4 \text { anos. } \\
\text { O diagnóstico mais frequente } \\
\text { foi de dor miofascial seguido } \\
\text { de dor neuropática. A } \\
\text { prevalência de sintomas de }\end{array}$ & $\begin{array}{l}\text { Este estudo revela uma alta } \\
\text { prevalência de sintomas de } \\
\text { depressão e ansiedade e } \\
\text { alterações no padrão do sono } \\
\text { em pacientes com dor crônica, } \\
\text { justificando a investigação }\end{array}$ \\
\hline
\end{tabular}




\begin{tabular}{|c|c|c|c|c|}
\hline & $\begin{array}{l}\text { depressão } \quad \text { em } \\
\text { pacientes com } \\
\text { dor crônica. }\end{array}$ & & $\begin{array}{l}\text { ansiedade foi } 72,8 \% \text {, de } \\
\text { depressão foi } 61,5 \% \text { e de } \\
\text { alteração do sono } 93 \% \text {. }\end{array}$ & $\begin{array}{l}\text { destes distúrbios neste grupo } \\
\text { de pacientes. }\end{array}$ \\
\hline $\begin{array}{l}\text { ANTUNES, R. S.; } \\
\text { MACEDO, B. } \\
\text { G.; AMARAL, T. } \\
\text { S.;GOMES, H. } \\
\text { A.;PEREIRA,L.S. } \\
\text { M.; ROCHA, F. } \\
\text { L. }\end{array}$ & 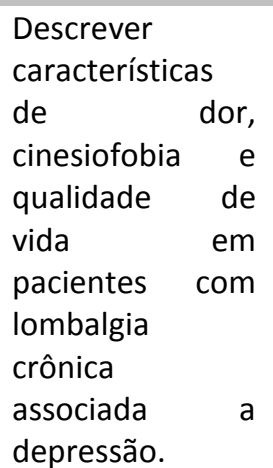 & 193 & $\begin{array}{l}\text { A prevalência de depressão } \\
\text { foi de } 32,1 \% \text {. O grupo com } \\
\text { depressão teve pior } \\
\text { pontuação com relação à dor, } \\
\text { cinesiofobia e qualidade de } \\
\text { vida (capacidade funcional, } \\
\text { aspectos físicos, dor, estado } \\
\text { geral de saúde, vitalidade, } \\
\text { aspectos sociais, aspectos } \\
\text { emocionais e saúde mental). }\end{array}$ & $\begin{array}{l}\text { Pacientes com lombalgia e } \\
\text { depressão apresentaram maior } \\
\text { intensidade de dor, maior } \\
\text { medo de movimento e pior } \\
\text { qualidade de vida. }\end{array}$ \\
\hline $\begin{array}{l}\text { SNEKKEVIK, H.; } \\
\text { ERIKSEN, H. R.; } \\
\text { TANGEN, T.; } \\
\text { CHALDER, T.; } \\
\text { REME, S. E. }\end{array}$ & $\begin{array}{l}\text { O objetivo do } \\
\text { estudo foi } \\
\text { examinar a } \\
\text { prevalência de } \\
\text { substancial } \\
\text { fadiga e a } \\
\text { associação entre } \\
\text { fadiga, } \\
\text { depressão e dor. }\end{array}$ & 569 & $\begin{array}{l}\text { Aqueles com substancial } \\
\text { fadiga tiveram dor de maior } \\
\text { intensidade, r maiores } \\
\text { sintomas depressivos e mais } \\
\text { incapacidade se comparado } \\
\text { aqueles sem } r \text { fadiga } \\
\text { substancial. } \\
\text { musculoesquelética } \\
\text { depressão } \\
\text { independentemente } \\
\text { associadas com substancial } \\
\text { fadiga. }\end{array}$ & $\begin{array}{l}\text { A grande maioria dos doentes } \\
\text { listados com lombalgia crônica } \\
\text { relataram uma fadiga } \\
\text { substancial. Aqueles com } \\
\text { fadiga tiveram mais dores e } \\
\text { sintomas depressivos e um } \\
\text { risco significante de terem } \\
\text { dificuldades motoras em } 3,6 \text { e } \\
12 \text { meses. }\end{array}$ \\
\hline $\begin{array}{l}\text { ELLEGAARD, H.; } \\
\text { PEDERSEN, B. } \\
\text { D. }\end{array}$ & $\begin{array}{l}\text { O objetivo foi } \\
\text { investigar como } \\
\text { alguns } \\
\text { tratamentos } \\
\text { influenciam a } \\
\text { capacidade do } \\
\text { paciente em } \\
\text { lidar com a dor } \\
\text { lombar crônica } \\
\text { quando } \\
\text { associado à } \\
\text { depressão. }\end{array}$ & 130 & $\begin{array}{l}\text { Em geral, a soma dos fatores } \\
\text { de estresse em conjunto com } \\
\text { depressão e dor foram } \\
\text { mostrados para provocar } \\
\text { sintomas de stress. O estresse } \\
\text { foi diminuído no tratamento } \\
\text { psicoterapêutico e recursos } \\
\text { internos foram } \\
\text { reestabelecida, que se } \\
\text { manifestam como maior } \\
\text { relaxamento, presença, } \\
\text { autoestima, responsabilidade } \\
\text { e felicidade. Este, por sua vez, } \\
\text { ajudou os pacientes a tratara } \\
\text { sua dor lombar crônica. }\end{array}$ & $\begin{array}{l}\text { Lombalgia crônica é um fator } \\
\text { de estresse por si só, mas } \\
\text { quando combinado com a } \\
\text { depressão pode ser } \\
\text { considerado como dois } \\
\text { complexos de sintomas que } \\
\text { afetam mutuamente entre si } \\
\text { de forma negativa. Neste } \\
\text { estudo, a terapia Gestalt e o } \\
\text { método-SE pode ter ajudado a } \\
\text { diminuir o nível de estresse dos } \\
\text { seus pacientes e restaurar seus } \\
\text { próprios recursos internos, } \\
\text { aumentando assim a sua } \\
\text { capacidade de lidar com a sua } \\
\text { dor lombar crônica. }\end{array}$ \\
\hline $\begin{array}{l}\text { REME, S. E.; } \\
\text { LIE, S. A.; } \\
\text { ERIKSEN, H. R. }\end{array}$ & $\begin{array}{l}\text { Examinar a } \\
\text { sensibilidade de } \\
2 \quad \text { perguntas } \\
\text { simples } \\
\text { comparados com } \\
2 \quad \text { longos } \\
\text { questionários } \\
\text { para aferição de } \\
\text { depressão e } \\
\text { ansiedade em } \\
\text { pacientes com } \\
\text { dor lombar } \\
\text { crônica. }\end{array}$ & 564 & $\begin{array}{l}\text { A prevalência de desordens } \\
\text { depressivas foi de } 4 \%(n=21) \text { e } \\
\text { a prevalência de sintomas de } \\
\text { ansiedade foi de } 12 \% \text { ( } n=69) \text {, } \\
\text { através do questionário MINI, } \\
\text { considerado o padrão ouro. O } \\
\text { questionário HADS mostrou } \\
18 \% \text { e } 23 \% \text { para depressão e } \\
\text { ansiedade, respectivamente. } \\
\text { Já o HSCL indicou a presença } \\
\text { de sintomas de depressão } \\
\text { em29\%e ansiedade em } 20 \% \text {. }\end{array}$ & $\begin{array}{l}\text { Os questionários menores } \\
\text { foram mais sensíveis para } \\
\text { depressão do que para } \\
\text { ansiedade. É necessário a } \\
\text { aplicação dos questionários } \\
\text { maiores em outras populações } \\
\text { para comparação de } \\
\text { resultados. }\end{array}$ \\
\hline $\begin{array}{l}\text { HINCAPIÉ, C. A.; } \\
\text { CASSIDY, J. D.; } \\
\text { CÔTÉ, P. }\end{array}$ & $\begin{array}{l}\text { Determinar se } \\
\text { uma história de } \\
\text { trabalho, } \\
\text { durante a vida, }\end{array}$ & 1086 & $\begin{array}{l}\text { Dos entrevistados, } 38 \% \\
\text { relataram uma história de dor } \\
\text { lombar relacionada ao } \\
\text { trabalho. } \quad \text { Possivelmente, }\end{array}$ & $\begin{array}{l}\text { A análise mostrou uma } \\
\text { associação entre episódios } \\
\text { passados de dor lombar } \\
\text { relacionada ao trabalho e }\end{array}$ \\
\hline
\end{tabular}




\begin{tabular}{|c|c|c|c|c|}
\hline & $\begin{array}{l}\text { que afete a } \\
\text { coluna lombar é } \\
\text { associado com a } \\
\text { prevalência e } \\
\text { severidade de } \\
\text { dores lombares, } \\
\text { sintomas } \\
\text { depressivos ou } \\
\text { ambos, na } \\
\text { população em } \\
\text { geral. }\end{array}$ & & $\begin{array}{l}\text { nesses pacientes a dor é } \\
\text { maior. Não houve associação } \\
\text { entre a história de injúria } \\
\text { lombar no trabalho com } \\
\text { sintomas depressivos. }\end{array}$ & $\begin{array}{l}\text { aumento da sua severidade, } \\
\text { mas não houve associação com } \\
\text { depressão. }\end{array}$ \\
\hline $\begin{array}{l}\text { HA, J. Y.; } \\
\text { KIM, E. S.; } \\
\text { KIM, H. J.; } \\
\text { PARK, S. J. }\end{array}$ & $\begin{array}{l}\text { Investigar } \\
\text { sintomas } \\
\text { depressivos e } \\
\text { seus fatores } \\
\text { relacionados a } \\
\text { pacientes com } \\
\text { dor lombar } \\
\text { crônica na } \\
\text { Coreia. }\end{array}$ & 1426 & $\begin{array}{l}\text { Cerca de } 26.0 \% \text { (371 } \\
\text { pacientes) mostraram-se com } \\
\text { sintomas depressivos. A maior } \\
\text { parte eram mulheres. } \\
\text { Também foi mostrado maior } \\
\text { prevalência de sintomas } \\
\text { depressivos conforme o maior } \\
\text { número de doenças crônicas } \\
\text { acumuladas } \\
\text { entrevistado. }\end{array}$ & $\begin{array}{l}\text { Este estudo mostra a } \\
\text { associação entre pacientes com } \\
\text { dor lombar crônica e sintomas } \\
\text { depressivos. } \\
\text { terapêuticas para tratamento } \\
\text { da patologia lombar deve } \\
\text { considerar o risco do } \\
\text { desenvolvimento de sintomas } \\
\text { depressivos. }\end{array}$ \\
\hline $\begin{array}{l}\text { FRACARO, G. } \\
\text { A.; } \\
\text { BERTOR, W. R. } \\
\text { R.; } \\
\text { SILVA, L. I.; } \\
\text { BRANDL, L.; } \\
\text { ZANINI, G. M.; }\end{array}$ & $\begin{array}{l}\text { O objetivo deste } \\
\text { estudo foi } \\
\text { comparar a } \\
\text { distância } \\
\text { percorrida no } \\
\text { teste de } \\
\text { caminhada de } \\
\text { seis minutos e as } \\
\text { variáveis } \\
\text { psicossociais. }\end{array}$ & 17 & $\begin{array}{l}\text { O grupo lombar apresentou } \\
\text { níveis de incapacidade } \\
\text { significativamente maiores } \\
\text { que o grupo controle(1,25 } \pm \\
2,1 \%) \text {. Também se observou } \\
\text { que o grupo lombar } \\
\text { apresentou níveis de tensão, } \\
\text { fadiga e confusão mental } \\
\text { significativamente mais alto } \\
\text { que o grupo controle. Não } \\
\text { houve diferença relevante de } \\
\text { sintomas depressivos entre os } \\
\text { grupos. }\end{array}$ & $\begin{array}{l}\text { Portadores de dor lombar } \\
\text { crônica não apresentaram } \\
\text { diferença, em relação aos seus } \\
\text { pares livres de dor, no } \\
\text { desempenho do TC6, no estado } \\
\text { de humor de depressão, de } \\
\text { raiva e de vigor. Contudo, } \\
\text { apresentaram maiores níveis } \\
\text { de incapacidade e piores } \\
\text { índices no estado de humor de } \\
\text { fadiga, de tensão e de confusão } \\
\text { mental. }\end{array}$ \\
\hline $\begin{array}{l}\text { SALVETTI, M. G; } \\
\text { PIMENTA, C. A. } \\
\text { M.; } \\
\text { BRAGA, P. E.; } \\
\text { CORRÊA, C. F. }\end{array}$ & $\begin{array}{l}\text { Identificar a } \\
\text { prevalência e os } \\
\text { fatores } \\
\text { associados à } \\
\text { incapacidade em } \\
\text { pacientes com } \\
\text { dor lombar } \\
\text { crônica. }\end{array}$ & 177 & $\begin{array}{l}\text { A prevalência de incapacidade } \\
\text { foi de } 65 \% \text { (IC95\%: } 57,5 \text { - } \\
72,0 \text { ) e era de moderada a } \\
\text { grave em } 80,7 \% \text { dos } \\
\text { pacientes. O modelo de } \\
\text { regressão múltipla identificou } \\
\text { três fatores } \\
\text { independentemente } \\
\text { associados à incapacidade: } \\
\text { ausência de trabalho } \\
\text { remunerado, auto eficácia } \\
\text { baixa e depressão. Os fatores } \\
\text { associados à incapacidade } \\
\text { identificados } \\
\text { modificáveis. }\end{array}$ & $\begin{array}{l}\text { Intervenções como recolocação } \\
\text { no trabalho, tratamento para a } \\
\text { depressão e reconceitualização } \\
\text { da crença de auto eficácia } \\
\text { podem ter um impacto } \\
\text { importante na prevenção e } \\
\text { redução de incapacidade. }\end{array}$ \\
\hline
\end{tabular}

Dos artigos incluídos na presente revisão, quatro foram desenvolvidos no Brasil e cinco em outros países, como Estados Unidos, Noruega e Coreia. Quanto ao nível de evidência, três correspondem ao nível 1 , cinco ao nível 2 e um ao nível 3. Dentre os estudos analisados sete foram desenvolvidos por médicos, um por enfermeiros e um por fisioterapeutas. Quanto às publicações, seis foram publicadas em revistas e arquivos médicos, uma em uma revista de enfermagem e duas em revistas multidisciplinares.

Para análise estatística, cinco trabalhos aceitam $p<5 \%$ como significativo e um $p<10 \%$. Os 
testes estatísticos utilizados foram qui-quadrado, teste T, T de Student e Mann-Whitney, dando destaque ao teste de qui-quadrado, utilizado em três artigos. Apenas um trabalho não descreveu a análise estatística utilizada.

Todos os trabalhos apresentaram o tamanho da amostra, a porcentagem de homens e mulheres participantes e a faixa etária estudada. Dos estudos incluídos nessa revisão, apenas um tinha maior participação masculina. Apesar de a maioria apresentar dados sobre nível socioeconômico, escolaridade e ocupação dos participantes, nenhum apresentou a raça dos mesmos.

Em sete estudos são apresentados às porcentagens dos participantes que tinham sintomas depressivos. Os outros dois não obtiveram resultados que correlacionassem dor lombar crônica e depressão. Três estudos apresentam, além dos dados sobre depressão, informações sobre ansiedade, fadiga e distúrbios do sono.

Apenas um trabalho não apresentava o questionário, sobre depressão, utilizado na entrevista com os pacientes. Os demais aplicaram questionários validados para análise dos sintomas depressivos, com ênfase nos seguintes testes: Hospital Anxiety and Depression Scale (HA DS) e Beck Depression Inventory Scale (BDIS).

Os artigos selecionados para a revisão apresentam revisão bibliográfica, objetivos claros, conclusões que respondem aos objetivos e utilização de gráficos e tabelas para elucidação dos resultados.

\section{DISCUSSÃO}

A amostra total de todos os estudos soma 4562 participantes, maioria feminina e média de idade de 45 anos. Todos estes com relato de dor lombar crônica. Em oito estudos não foram analisados as causas das dores dos entrevistados. Apenas um estudo associou a cronificação das dores com episódios passados inerentes à ocupação do paciente. Dessa forma, pode-se inferir que nem todos os quadros álgicos são decorrentes da mesma causa, o que poderia interferir nos resultados das pesquisas.

Sete estudos comprovaram a existência da relação entre lombalgia crônica e depressão. Nesses estudos podem-se encontrar resultados que variam de $4 \%$ a $69,7 \%$, com média de $30,51 \%$. Essa variação dos resultados pode ser associada ao uso de diferentes questionários para coleta de dados $\mathrm{e}$ as diferentes amostras utilizadas, já que estas não obedeceram a critérios de inclusão semelhantes. Dois estudos não encontraram correlação entre depressão e lombalgia crônica, mas um deles encontrou correlação com ansiedade e o outro com aumento da fadiga.

Os trabalhos foram desenvolvidos em diferentes países, o que representa diferentes amostras. Apesar disso, a maioria dos estudos comprovaram a associação entre dor lombar crônica e depressão, o que indica que mesmo com amostras e critérios de inclusão diferentes, existe uma correlação real entre essas condições.

Três trabalhos avaliaram os níveis de ansiedade, onde os resultados encontrados variaram entre $12 \%$ e $72,9 \%$. Um trabalho analisou a fadiga e outros distúrbios do sono, com valores percentuais de, respectivamente, $69,7 \%$ e $93 \%$.

\section{CONCLUSÃO}

Concluindo a presente revisão integrativa percebe-se que existem trabalhos que comprovam a associação entre dor lombar crônica e depressão, porém não foi analisada coerentemente a causa da lombalgia.

Devido a grande incidência, novas pesquisas devem ser iniciadas para o tratamento adequado dos pacientes com dor lombar crônica associada aos quadros depressivos e de ansiedade, possibilitando ao paciente ter uma melhor qualidade de vida.

A promoção de saúde e prevenção da instalação das patologias crônicas de coluna, através de medidas simples de educação da população é a melhor forma de prevenção da instalação do quadro crônico. E mesmo após a instalação do quadro crônico, é de extrema importância o tratamento precoce para que não haja desenvolvimento de patologias agravantes, como a depressão.

\section{REFERÊNCIAS}

1- Ferreira M S, Navega M T. Efeitos de um programa de orientação para adultos com lombalgia. Acta ortop. bras. 2010 ; 18 ( 3 ): 127131. DOI: http://dx.doi.org/10.1590/S141378522010000300002.

2 - Rached RDVA, Rosa CDP, Alfieri FM, Amaro SMC, Nogueira B, Dotta L et al . Lombalgia inespecífica crônica: reabilitação. Rev. Assoc. Med. Bras. [Internet]. 2013 Dec; 59( 6 ): 536- 
553. DOI:

http://dx.doi.org/10.1016/i.ramb.2013.10.003.

3- Andersson GBJ. Epidemiological

features of chronic low-back pain. Lancet. 1999

Aug 14;354(9178):581-5. DOI: 10.1016/S0140-

6736(99)01312-4.

4- Ford J, Story I, Sullivan PBO, McMeeken J. Classifications systems for low back pain: A review of the methodology for development and validation. Physical Therapy Reviews. 2014 Jan; 12(1):33-42. DOI: 10.1179/108331907X174961 5- Johannes CB, Le TK, Zhou X, Johnston

$\mathrm{JA}$, Dworkin RH..

The prevalence of chronic pain in United States adults: results of an internetbased survey. J Pain. 2010 Nov;11(11):1230-9. DOI: 10.1016/j.jpain.2010.07.002

6- Silva MC, Fassa AG, Valle NCJ. Dor lombar crônica em uma população adulta do Sul do Brasil: prevalência e fatores associados. Cad. Saúde Pública . 2004 Apr; 20( 2 ): 377-385. DOI: http://dx.doi.org/10.1590/S0102311X2004000200005.

7- Pontes H. A incidência de lombalgia em indústria de fundição: um estudo de caso sob a ótica da ergonomia [dissertação]. Ponta Grossa: Universidade Tecnológica Federal do Paraná, 2005.

8- Tavafian SS, Jamshidi A, Mohammad K, Montazeri A. Low back pain education and short term quality of life: a randomized trial. BMC Musculoskeletal Disorders 2007, 8:21.

DOI:10.1186/1471-2474-8-21.

9- Malateaux JM, Ricci FR, Fragoso YD. Investigation of low back pain in a non-hospital population of the coastline of the State of São Paulo. Rev. dor . 2011 Mar; 12( 1 ): 19-22. DOI: http://dx.doi.org/10.1590/S1806-

\section{5}

10- Pignone MP, Gaynes BN, Rushton JL, Burchell $\mathrm{CM}$, Orleans CT, Mulrow CD, Lohr KN. Screening for depression in adults: a summary of the evidence for the U.S. Preventive Services Task Force. Ann Intern Med. 2002 May 21;136(10):765-76. http://dx.doi.org/10.7326/0003-4819-136-10200205210-00013

11- Haggman S, Maher CG, Refushauge CM. Screening for symptoms of depression by physical therapists managing low back pain. Physical Therapy. 2004 Dec; 84(12):1157-1166. 12- Marques-Vieira CMA, Sousa LMM, Carias JFMM, Caldeira SMA. Nursing diagnosis "impaired walking" in elderly patients: integrative literature review. Rev. Gaúcha Enferm. 2015 Mar; 36( 1 ): 104-111. DOI: http://dx.doi.org/10.1590/19831447.2015.01.48602.

13- Crossetti, MGO. Revisão integrativa de pesquisa na enfermagem o rigor cientifico que Ihe é exigido [editorial]. Rev Gaúcha Enferm., Porto Alegre (RS) 2012 jun;33(2):8-9.

14- URSI, Elizabeth Silva ; GAVÃO, Cristina Maria. Prevenção de lesões de pele no perioperatório: revisão integrativa da literatura. Rev LatinoAm. Enfermagem. 2006 Feb; 14(1):124-131.

Recebido para publicação em 31/08/2015

Revisado em 10/09/2015

Aceito em 15/09/2015 